\title{
Molecular Mechanisms by which GLP-1 RA and DPP-4i Induce Insulin Sensitivity
}

Running Title: GLP-1RA and Insulin Resistance

\author{
Habib Yaribeygi ${ }^{1 *}$, Thozhukat Sathyapalan², Amirhossein Sahebkar ${ }^{3,4,5 *}$
}

\author{
${ }^{1}$ Research Center of Physiology, Semnan University of Medical Sciences, Semnan, Iran \\ ${ }^{2}$ Academic Diabetes, Endocrinology and Metabolism, Hull York Medical School, \\ University of Hull \\ ${ }^{3}$ Biotechnology Research Center, Pharmaceutical Technology Institute, Mashhad University \\ of Medical Sciences, Mashhad, Iran \\ ${ }^{4}$ Neurogenic Inflammation Research Center, Mashhad University of Medical Sciences, \\ Mashhad, \\ Iran \\ ${ }^{5}$ School of Pharmacy, Mashhad University of Medical Sciences, Mashhad,
}

\section{*Corresponding Author}

Amirhossein Sahebkar, Biotechnology Research Center, Mashhad University of Medical Sciences, Mashhad 9177948564, Iran. Tel: +985138002299; Fax: +985138002287; E-mail:

sahebkara@mums.ac.ir; amir_saheb2000@yahoo.com 


\begin{abstract}
Glucagon-like peptide- 1 is a peptide of incretin family which is used in the management of diabetes as glucagon-like peptide-1 receptor agonist (GLP-1RA). Dipeptidyl peptidase-4 enzyme metabolizes glucagon-like peptide- 1 and various dipeptidyl peptidase-4 enzyme inhibitors (DPP-4i) are also used in the management of diabetes. These antidiabetic agents provide anti-hyperglycemic effects via several molecular mechanisms including promoting insulin secretion, suppression of glucagon secretion and slowing the gastric emptying. There is some research suggesting that they can induce insulin sensitivity in peripheral tissues. In this study, we review the possible molecular mechanisms by which GLP-1RA and DPP-4i can improve insulin resistance and increase insulin sensitivity in insulin-dependent peripheral tissues.
\end{abstract}

Keywords: Glucagon-like peptide-1, Glucagon-like peptide-1 receptor agonist, dipeptidyl peptidase-4 inhibitors, Oxidative Stress, GLUT-4, Diabetes Mellitus. 


\section{Introduction}

The incidence of diabetes mellitus (DM) is increasing worldwide in an epidemic proportion [1]. This chronic disorder affects several metabolic pathways resulting in the production of various toxic byproducts leading to various complications of diabetes [2-4]. DM acts as a potent upstream event for many pathophysiologic pathways including oxidative stress, inflammation, fibrosis, apoptotic processes, TLR (toll-like receptor) activation, necrotic events and the activity of death receptors thereby inducing various forms of tissue dysfunction and diabetic complications [4-6]. Inadequate response to circulatory insulin, which is known as insulin resistance, play significant roles in the onset and progress of these pathologic pathways [7-9]. Therefore, different classes of antidiabetic medicines have been developed to increase the tissue sensitivity to insulin and normalize the glycemia thereby preventing the pathophysiologic mechanisms involved in diabetic complications [10-12].

Glucagon-like peptide receptor-1 agonists (GLP-1RA) is one of the classes of antidiabetic medications which provide anti-hyperglycemic effects via several molecular mechanisms [13]. These pharmacologic agents exert their pleiotropic effects by lowering postprandial hyperglycemia mainly via binding to their specific receptors in various tissues [13]. They have a lower risk of hypoglycemia due to their glucose-dependent mode of action [14, 15]. Dipeptidyl peptidase-4 inhibitors (DPP-4i) are another group of antidiabetic agents which work by inhibition of GLP-1 breakdown thereby increasing its bioavailability [13]. There is some evidence suggesting that they also can increase insulin sensitivity in insulin-dependent peripheral tissues [16-18]. In the current review, we present the possible molecular mechanisms by which GLP-1RA modulate insulin sensitivity in patients with diabetes. It must be noted that higher levels of GLP-1 modulate glucose homeostasis by several mechanisms including slowing gastric emptying, inhibiting gluconeogenesis and suppression of appetite [19, 20]. In this review, we focus on its insulin-sensitizing effects. 


\section{Classification of Diabetes Mellitus}

Type 1 (T1DM) and type 2 diabetes (T2DM) are the two common forms of diabetes [21]. (T1DM accounts for about 5-10\% of all patients with diabetes and mainly results from betacell dysfunction, reduction in insulin release and lower circulatory levels of insulin [21]. Type 2 diabetes (T2DM) is the most prevalent form of diabetes which account for more than $90 \%$ of patients with diabetes [21]. T2DM mainly results from an inadequate cellular response to insulin (insulin resistance) in insulin-dependent peripheral tissues in addition of beta-cell dysfunction and other pathophysiological defects [21]. Gestational diabetes is another type of DM which occurs in pregnant women likely due to hormonal variations during pregnancy period and insulin resistance [22]. Other forms of diabetes are latent autoimmune diabetes in adults or LADA, maturity-onset diabetes of the young (MODY), secondary diabetes to various conditions such as pancreatitis and secondary to various medications such as corticosteroids [23].

\section{Glucose Homeostasis and Insulin Signaling}

Glucose is a hexose carbohydrate and the preferred metabolic substrate for many types of human cells. It is a hydrophilic molecule with a molecular weight of 180 and thereby, is too big for easily passing across the cellular membrane [24, 25]. The two major pathways for glucose to enter cells are via active transporters (by sodium-glucose cotransporters or SGLTs) or via specific carriers (by glucose transporters or GLUT) [25]. GLUTs are a family of proteins that provide bidirectional facilitated glucose transport across the cell membrane without consuming energy and are based on the glucose concentration gradient across the cell membrane [25, 26]. These are at least 14 different GLUT proteins in human of which GLUT14 are more important in glucose homeostasis [25, 27]. GLUT-4 works completely dependent on insulin hormone $[25,28]$. It remains in the cytoplasmic vesicles in non-active forms and 
activates in response to insulin and translocate into the specific cell membrane and thereby facilitates glucose entering into the adipocytes, cardiac and skeletal muscle cells [25, 27]. Since striated muscle tissues act as a storage for glucose as glycogen, GLUT-4 plays an important role in the whole-body glucose homeostasis [27].

Insulin is an important metabolic hormone made up of 51 amino acids and has a molecular weight of 5808D. It belongs to a family of peptides which includes insulin-like growth factors (IGF) I and II, relaxin and some other insulin-like peptides [29, 30]. It has two distinct chains known as $\alpha$ and $\beta$. These chains are linked together by two disulfide bridges and a third one which is within the $\alpha$ chain [29, 30]. Insulin is secreted by pancreatic beta-cells when glucose enters into the islets via specific glucose carriers known as GLUT-2 by facilitated diffusion in responses to various stimuli such as higher plasma levels of glucose and amino acids [29, 30].

Glucose is phosphorylated to G6P (glucose-6-phosphate) within the beta-cells [31]. This process is catalyzed by the glucokinase enzyme activity which acts as "glucose sensor" into the islets and thereby controls the rate of glucose entering into the islets and in turn the rate of insulin release [31]. G6P increases the ATP (adenosine triphosphate) to ADP (adenosine diphosphate) ratio in the beta-cells which in turns closes the ATP-sensitive $\mathrm{K}^{+}$channels, depolarizes beta-cell membrane and augments the intracellular calcium levels by opening the voltage-dependent $\mathrm{Ca}^{2+}$ channels in the pancreatic beta-cells [31]. Increasing the intracellular $\mathrm{Ca}^{2+}$ levels induces the exocytosis of secretory granules containing insulin/proinsulin molecules from the beta-cells into the circulation [31].

Insulin facilitates the glucose entering into the adipocytes, cardiac and skeletal muscle cells by sequential steps known as insulin signal transduction (IST) [32]. IST is initiated by binding the insulin to the $\alpha$ chain of specific receptors known as insulin receptors (IRs). IR is a member of transmembrane tyrosine kinases composed of $\alpha$ and $\beta$ which is activated by insulin as well as 
by IGF ${ }^{1} 1$ and 2 [32]. This process induces structural changes in $\beta$ chain domain by prompting the auto-phosphorylation of tyrosine residues followed by downstream events such as the recruitment of different adaptor proteins i.e. insulin receptor substrates (IRSs), $\mathrm{Shc}^{2}$ protein, and APS protein ${ }^{3}[25,33]$. These processes provide an appropriate binding site for the IRS- $1^{4}$ [33]. IRS-1 can be also activated by other kinases including ERK1/2 ${ }^{5}$, atypical $\mathrm{PKC}^{6}, \mathrm{~S} 6 \mathrm{~K} 1^{7}$, $\mathrm{SIK}^{8}{ }^{8}$ AKT, $\mathrm{mTOR}^{9}$ and ROCK $1^{10}$ as well as other types of kinases such as AMPK ${ }^{11}$ and GSK3 $^{12}[33,34]$. Activated IRS-1 binds to PI3K ${ }^{13}$ and activate it which in turn catalyzes the conversion of $\mathrm{PIP}_{2}{ }^{14}$ to $\mathrm{PIP}_{3}{ }^{15}$ [35]. $\mathrm{PIP}_{3}$ is itself a potent activator for $\mathrm{PKB}$ (protein kinase $\mathrm{B}$ also known as Akt) [35]. Akt is final effector in this pathway which facilitates glucose entering into the cells by localization of GLUT-4 and inhibiting the glycogen synthase kinase leading to more glycogen synthesis $[30,35]$. The complicated process of IST and insulin sensitivity can be modulated in all the above steps [36-38].

\section{GLP-1 Receptor Agonists and DPP-4 Inhibitors}

The glucagon-like peptide (GLP) is a protein belonging to the incretin family which is secreted from the intestine in response to food ingestion and stimulates the GLP-1 receptor (GLP-1R) [39]. The GLP-1R is a G-protein coupled protein mainly found in the pancreatic beta-cells and is involved in regulating the blood glucose in several ways [40]. It composed of two domains,

\footnotetext{
${ }^{1}$ Insulin-like growth factor

2 SHC-transforming

${ }^{3}$ adapter protein with a $\mathrm{PH}$ and $\mathrm{SH} 2$ domain

${ }^{4}$ insulin receptor substrate type 1

${ }^{5}$ extracellular signal-regulated kinase 1/2

${ }^{6}$ protein kinase $\mathrm{C}$

${ }^{7}$ Ribosomal protein S6 kinase beta-1

${ }^{8}$ serine/threonine-protein kinase 2

${ }^{9}$ mammalian target of rapamycin

${ }^{10}$ Rho-associated protein kinase 1

${ }^{11}$ AMP-activated protein kinase

12 Glycogen synthase kinase 3

${ }^{13}$ phosphoinositide 3-kinase

${ }^{14}$ Phosphatidylinositol 4,5-bisphosphate

${ }^{15}$ Phosphatidylinositol 3,4,5-trisphosphate
} 
the extracellular domain binds the C-terminal helix of GLP-1 and the transmembrane domain binds to the N-terminal region of GLP-1 [41-44].

GLP-1 RA is a family of antidiabetic agents that mimic the effects of incretin hormone [4547]. Incretin is a family of metabolic hormones including GLP-1 and GIP (gastric inhibitory peptide), which decrease postprandial blood glucose by inhibition of glucagon secretion from pancreatic $\alpha$-cells and inducing insulin release from $\beta$-cell in a blood glucose-dependent manner [46, 48, 49]. GLP-1RA have additional effects such as delaying the gastric emptying, appetite suppression, declining nutrient absorption in the gut, improvement of lipid metabolism, inhibition of pancreatic $\beta$-cell apoptosis and induction of beta-cell neogenesis [48, $50,51]$.

Table 1. Two main classes of antidiabetic drugs which work by GLP-1 level

\begin{tabular}{|c|l|l|c|}
\hline $\begin{array}{c}\text { Classes of } \\
\text { Antidiabetic Drug }\end{array}$ & \multicolumn{1}{|c|}{ Approved Forms } & $\begin{array}{l}\text { Mechanisms of Hypoglycemic } \\
\text { Effects }\end{array}$ & Ref. \\
\hline GLP-1RA & $\begin{array}{l}\text { Exenatide, Albiglutide, } \\
\text { Liraglutide, Lixisenatide } \\
\text { Semaglutide, } \\
\text { Dulaglutide }\end{array}$ & $\begin{array}{l}\text { Mimic the hypoglycemic effects } \\
\text { of incretin such as insulin } \\
\text { secretion, glucagon release } \\
\text { suppression, appetite inhibition, } \\
\text { slowing the gastric emptying }\end{array}$ & [46, 47] \\
\hline DPP-4i & $\begin{array}{l}\text { Sitagliptin, Saxagliptin, } \\
\text { Vildagliptin, Linagliptin }\end{array}$ & $\begin{array}{l}\text { enzyme (DPP-4 enzyme) } \\
\text { leading to enhanced } \\
\text { hypoglycemic effects of native } \\
\text { GLP-1 }\end{array}$ & [52, 53] \\
\hline
\end{tabular}

Dipeptidyl peptidase-4 inhibitor (DPP-4i) are another type of antidiabetic agents with similar hypoglycemic effects to GLP-1RA. They act by preventing the inactivation of GLP-1 thereby increasing the active levels of native GLP-1 $[52,53]$. After posttranslational processes of preglucagon (PG) peptides in intestinal L cells, at least four separate forms of PG produced that are all can be inactivated by DPP-4 enzyme by removing the two amino acids from $\mathrm{N}$-terminal residue [54]. DPP-4 is mainly located on the surface of the most types of cells including the 
endothelial cells, renal epithelial cells and T lymphoid cells, where they have a binding partner element which makes intracellular signals [55].

\section{GLP-1 and Insulin Sensitivity}

In addition to the aforementioned hypoglycemic effects, there is strong evidence indicating GLP-1 increases insulin sensitivity in peripheral tissues [17, 19] (fig 1). The higher levels of GLP-1, by either GLP-1RA and/or DPP-4i administration, can induce peripheral insulin sensitivity through several direct or indirect molecular pathways $[17,18,56]$. In the following paragraphs, we discuss the possible molecular mechanisms involved in the GLP-1 dependent insulin sensitivity.

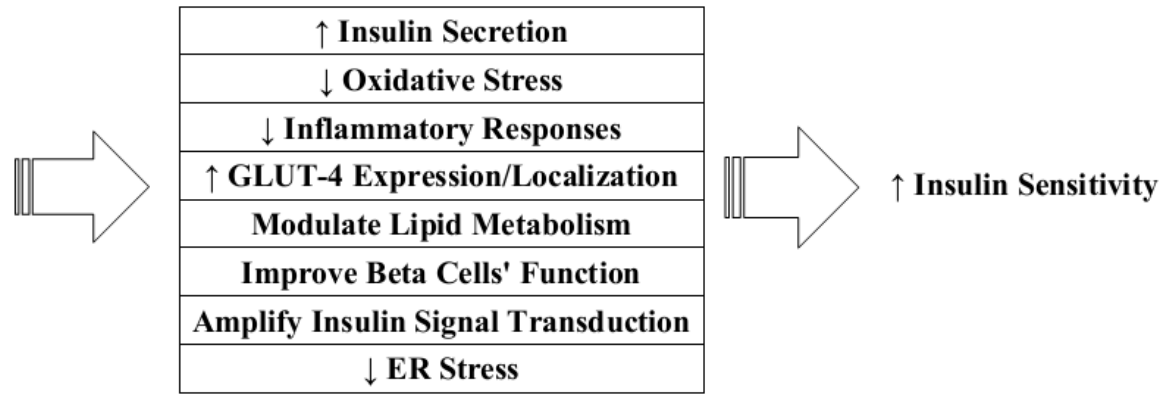

Figure 1: Possible molecular mechanisms by which GLP-1 induces insulin sensitivity

\section{GLP-1 and Insulin Secretion}

GLP-1 receptors analogues and/or its breakdown enzyme inhibitors are known stimulators for pancreatic beta cells to secrete postprandial insulin in response to higher levels of blood glucose in a concentration-dependent manner [57]. It has suggested that GLP-1 induces insulin secretion through several molecular pathways such as cAMP production, $\mathrm{Ca}^{2+}$ dependent voltage-gated channels, proinsulin granule recruitment and promoting vesicle ducking [57]. There is also evidence that DPP-4i can stimulate insulin release [58]. Consequently, GLP-1 based therapies are now routinely used in clinical practice for the treatment of T2DM and 
obesity $[57,59,60]$. This islet cell stimulating effect of GLP-1 could be one of the therapeutic targets even in some cases of T1DM and/or T2DM with dysfunctional beta-cells [57, 61, 62].

\section{GLP-1 and Oxidative Stress}

Oxidative stress has pivotal roles in the pathophysiology of many complications of diabetes as well as insulin resistance [63-65]. The free radical overload reduces the insulin sensitivity and induces insulin resistance by several molecular pathways including $\mathrm{IKK} \beta^{16} / \mathrm{NF}-\kappa B^{17}$ and $\mathrm{JNK}^{18}$ pathways, IRS degradation, GLUT-4 down-regulation, lowering the insulin-dependent IRS-1 and PIP-kinase transfer between cytoplasm and microsomes [65-68]. Hence, readjusting the redox state in favor of physiologic milieu with a balance between free radicals and antioxidative elements is an attractive goal for designing novel antidiabetic agents [11, 69, 70].

On the other hand, emerging evidence suggests that GLP-1 receptor induction by either GLP1RA and /r DPP-4i can result in improved antioxidative outcomes [39, 71, 72]. Oh and colleagues in 2017 suggested that GLP-1 have antioxidative potentials either by the glucoselowering effects or via Nrf2 ${ }^{19}$ signaling pathways and potentiation of antioxidant defense system [39, 73]. The inducers of Nrf2 signaling pathways improves insulin sensitivity in diabetic milieu [74, 75]. Deng and coworkers in 2018 showed that GLP-1 can induce Nrf2 signaling pathways and increases the expression of antioxidant elements in the neuronal cells [73]. Fernández-Millán and coworkers in 2016 found that GLP-1 directly increases Nrf2 signaling and improves oxidative stress in the pancreatic beta-cells [76]. Also, Puddu et al in 2013 provided further data suggesting GLP-1 attenuated the oxidative stress by suppressing $\mathrm{AGE}^{20}-\mathrm{RAGE}^{21}$ interaction in the diabetic milieu [77]. Moreover, Tomas et al in 2011

\footnotetext{
16 inhibitor of nuclear factor kappa-B kinase subunit beta

17 nuclear factor kappa-B

18 c-Jun $\mathrm{N}$-terminal kinase

${ }^{19}$ Nuclear factor erythroid 2-related factor 2

${ }^{20}$ Advanced glycation end product

${ }^{21}$ Receptors for AGE
} 
discovered a nano-peptide derived from GLP-1 which reduces the oxidative stress via mitochondria-dependent mechanism [78]. Patel et al in 2013 demonstrated that GLP-1 improves insulin sensitivity by attenuating the oxidative stress in diabetic mice [79]. Okada et al in 2014 and Rizzo et al in 2015 provided clinical evidence indicating liraglutide decreased the oxidative stress in patients with T2DM $[80,81]$. This evidence strongly suggested that GLP-1 can induce insulin sensitivity by ameliorating oxidative stress [77-81].

\section{GLP-1 and Inflammatory responses}

Inflammatory processes have pivotal roles in the pathophysiology of insulin resistance [65, 82]. These biologic events can impair IST via several molecular mechanisms including IKK $\beta / \mathrm{NF}-\kappa \mathrm{B}$ and $\mathrm{JNK}^{22}$ pathway activation, serine phosphorylation of IRS-1 in the site of 307, GLUT-4 and IRS-1 down-regulation as well as IRS-1 degradation [65, 83-85]. Hence, ameliorating the inflammation is one of the ideal therapeutic targets for antidiabetic medicines $[10,12]$. There is strong evidence indicating GLP-1 has potent anti-inflammatory potentials which may be beneficial against inflammatory responses and reduces the circulating inflammatory mediators leading to an increase in peripheral insulin sensitivity [12, 86-91]. Kodera et al in 2011 demonstrated that exendin-4, of the GLP-1RA, directly reduced inflammatory mediators such as ICAM (intercellular adhesion molecule-1), type IV collagen, and Nf-kb activation as well as reduced macrophage infiltration [91]. Also, Shiraki and coworkers in 2012 reported that GLP-1 attenuated inflammatory responses via lowering the TNF- $\alpha$ and Nf-kb expression levels in the umbilical endothelial cells [90]. Krasner et al in 2014 reported similar findings implying GLP-1 reduces inflammatory responses by calcium and AMPK dependent molecular pathways [87].

\footnotetext{
${ }^{22}$ c-Jun N-terminal kinase
} 
Guo and colleagues in 2016 found that exendin-4 improved insulin sensitivity by lowering the inflammatory responses [17]. They demonstrated that it markedly reduced macrophage-derived insulin resistance by Nf-kb dependent molecular pathway leading to lower inflammationinduced insulin resistance [17]. Also, Zheng et al in 2018 revealed that raising the levels of GLP-1 by DPP-4i improved insulin sensitivity by ameliorating the inflammatory responses via AMPK/mTOR molecular mechanisms in the diabetic animals [92]. Zhuge and coworkers in 2016 provided data indicating linagliptin improved insulin sensitivity by modulating macrophage-dependent inflammatory events [93]. These evidence suggest that GLP-1 can promote insulin sensitivity by a reduction in inflammatory responses [17, 92, 93].

\section{GLUT-4 Expression and Glucose Transport}

The GLUT-4 is insulin-dependent glucose carrier and any disruption of its function will result in insulin resistance $[94,95]$. There is some evidence that GLP-1 induces glucose transport across the cell membrane [96-98] and also induce GLUT-4 expression [99, 100]. Wang et al in 1997 demonstrated that GLP-1 increases GLUT-4 expression and glucose transport in adipocytes [100]. Villanueva-Peñacarrillo et al in 2001 reported that GLP-1 up-regulated the GLUT-4 expression at both mRNA and protein levels in adipocytes and muscle tissues of diabetic animals [99]. Also, Green and coworkers in 2012 observed that GLP-1 increased the GLUT-4 expression at the protein level by PI3-K dependent mechanism in human satellite cells [101]. Moreover, Andreozzi and coworkers in 2016 found that GLP-1 increased glucose transport across skeletal muscle cells via up-regulating the GLUT-4 by an AMPK-dependent manner [102]. Similarly, Li et al in 2014 demonstrated that liraglutide induces GLUT-4 translocation/expression by AMPK dependent mechanisms in skeletal muscles of diabetic mice [103]. Giannocco et al in 2013 provided similar data for DPP-4i indicating sitagliptin increased GLUT-4 translocation in the heart and skeletal muscle of rats [104]. This evidence strongly 
suggests that GLP-1 can improve insulin sensitivity by GLUT-4 expression/translocation [101104].

\section{GLP-1 and Plasma Lipids' Profile}

The plasma lipid profile affects insulin sensitivity [105, 106]. Emerging evidence has demonstrated the molecular relationships between lipid profile and dysfunctional lipid metabolism with varying degrees of insulin resistance indicating an optimum profile of plasma lipid is needed for physiological insulin sensitivity in peripheral tissues [106]. Higher levels of adipocytes and lipids/lipoproteins (e.g. cholesterol, triglycerides, HDL, VLDL and LDL), initiate molecular mechanisms that have a negative effect on insulin sensitivity [106-109].

GLP-1 can potentially modulate lipid metabolism and correct dyslipidemia through various molecular mechanisms and through activating different mediators and metabolic enzymes [110-112]. They can potentially modulate some microRNAs (miR) involved in lipid metabolism such as miR-200b, miR-200c, miR-34a, miR-338 and miR-21 [113-116]. Therefore, recent evidence suggested these hypoglycemic medications are promising therapeutic agents for dyslipidemia-induced disorders such as NAFLD (non-alcoholic fatty liver disease) and atherosclerosis in the diabetic milieu [117-120]. Ejarque et al in 2019 demonstrated that GLP-1 expressed on adipocytes regulates the adipose tissue metabolism leading to higher insulin sensitivity [121]. Also, Parlevliet and coworkers in 2012 found that GLP-1 receptor agonist, exendin-4, improved insulin sensitivity by regulating the VLDL production in diabetic mice [122]. Moreover, Cani et al in 2006 suggested that lipid-lowering and insulin-sensitizing effects of oligofructose are mediated via GLP-1 receptors in mice with T1DM [123]. There is a similar finding of the role of DPP-4i [124-126]. Baumeier et al in 2017 reported that higher expression levels of DPP-4i improved insulin sensitivity by readjusting the lipid metabolism [124]. Also, Silva and colleagues in 2019 showed that DPP-4i activity is correlated to the improvement of lipid metabolism and an increase in insulin sensitivity in 
patients with T2DM [125]. This evidence strongly suggested that GLP-1 can improve insulin sensitivity by controlling lipid metabolism and thereby improving dyslipidemia, however, more clinical studies are still needed.

\section{GLP-1 and Insulin Signaling Pathways}

The GLP-1 amplifies IST in various steps [127]. Wang et al in 1997 demonstrated that GLP-1 analogues improved insulin signaling in 3T3-L1 adipocytes [100]. Gao et al in 2007 provided evidence suggesting GLP-1 induce insulin signaling pathways by up-regulation of phosphorylated IR- $\beta$, IRS-1, Akt and GSK-3beta (glycogen synthase kinase 3 beta) in adipocytes leading to more insulin sensitivity [127]. Kawamori and coworkers in 2017 reported that GLP-1 signaling compensated IST impairment in the diabetic milieu and improved it [128]. They found that GLP-1 promoted Akt phosphorylation and protein expression of cyclins A, D1 and E leading to improved IST in adipocytes [128]. This evidence strongly suggested that GLP-1 has potent effects on insulin signaling pathways and thereby induces insulin sensitivity at least partly via IST induction [100, 127, 128].

\section{GLP-1 and Beta-cell Function}

A healthy beta- cell with normal physiologic function is a key element for appropriate insulin sensitivity [129, 130]. Many cases of DM are related to varying degrees of beta-cell dysfunction and thereby, reversing the pathophysiologic pathways involved in beta-cell dysfunction is an attractive target for the management of diabetes [131, 132]. There is some evidence indicating that GLP-1 can improve beta-cell function [133-136]. Tews et al in 2009 demonstrated that exendin-4 prevents beta-cell apoptosis and improves islets' function in a diabetic milieu [133]. Kawamori et al in 2017 showed that GLP-1 signaling induced beta-cell proliferation in adipocytes [128]. Caporarello and coworkers in 2017 reported that GLP-1 receptor agonists markedly enhanced pancreatic beta-cell generation in mice [137]. Also, Kim and colleagues in 2017 reported the same findings implying exendin-4 ameliorates beta-cell 
damage and improves insulin sensitivity in rats [134]. Moreover, Shimoda et al in 2011 found that liraglutide promoted pancreatic beta-cell function in diabetic mice [135]. Gedulin et al in 2005 reported that exendin-4 increases insulin sensitivity index and reduces blood glucose levels by increasing beta-cell mass in obese rats [136]. They showed that GLP-1 has trophic effects on pancreatic islets and exerts neogenesis and cell proliferation independent of glucose homeostasis and body weight $[136,138]$. Other possible pathways are also suggested [139, 140]. Hao et al in 2017 demonstrated that GLP-1 increases insulin sensitivity by improvement in beta-cell function through PDX-123/JAK signaling pathways [139]. Also, Zummo and coworkers in 2017 found that GLP-1 protects pancreatic beta-cell function by readjusting the autophagy/lysosomal homeostasis in INS-1E $\beta$-cells [140]. Akarte and colleagues in 2012 showed that DPP-4i (vidagliptin) increases beta-cell mass in the diabetic milieu [141]. This evidence suggest that improvement in beta-cell function is another pathway by which GLP-1 induces insulin sensitivity [133-136] [139].

\section{GLP-1 and ER Stress}

Endoplasmic reticulum (ER) is one of the main organelles in eukaryotic cells which have significant roles in the cellular homeostasis [142]. Various pathophysiological states and pharmacologic agents disturb the functioning of ER resulting in ER stress. ER stress contributes to the development of diabetes complications [143, 144]. There is some evidence suggesting GLP-1 can potentially improve ER stress in diabetic milieu [18, 135, 145]. Jiang et al in 2018 demonstrated that GLP-1 improved insulin sensitivity by ameliorating ER stress through mTOR signaling dependent pathways and its downstream mechanisms in adipocytes [18]. Also, Shimoda et al in 2011 reported that liraglutide improved ER stress-induced insulin resistance in the diabetic mice [135]. Moreover, Shimizu et al in 2012 found that vildagliptin (DPP-4i) improved ER stress in beta-cells by down-regulating the C/EBPB protein (a

\footnotetext{
${ }^{23}$ duodenal homeobox-1
} 
transcription factor) in diabetic mice [145]. This evidence suggest that GLP-1 can modulate

ER stress-induced insulin resistance [18, 135, 145].

Table 2. Possible insulin sensitizer influences of GLP-1 (Nrf2= Nuclear factor erythroid 2-related factor 2; HO-1= heme oxigenase-1; AGE= Advanced glycation end product; RAGE= receptor for AGE; Nf$\kappa b=$ nuclear factor kappa $b$; IRS-1=insulin receptor substrate-1; $E R=$ endoplasmic reticulum; mTOR= mammalian target of rapamycin; $\mathrm{C} / \mathrm{EBPB}=\mathrm{a}$ transcription factor)

\begin{tabular}{|c|c|c|}
\hline Molecular Mechanisms & Effects & Ref. \\
\hline Insulin Secretion & $\begin{array}{l}\text { Induces insulin expression/release via vary } \\
\text { molecular mechanisms such as cAMP } \\
\text { production, } \mathrm{Ca}^{2+} \text { dependent voltage-gated } \\
\text { channels, proinsulin granule recruitment and } \\
\text { promoting vesicle ducking }\end{array}$ & {$[57,59,60]$} \\
\hline Oxidative Stress & $\begin{array}{l}\text { Attenuates oxidative stress by several } \\
\text { molecular pathways such as Nrf2, HO- } 1 \text { and } \\
\text { AGE-RAGE interactions, reduces the free } \\
\text { radical generation }\end{array}$ & {$[39,73,77-81]$} \\
\hline $\begin{array}{l}\text { Inflammatory } \\
\text { Responses }\end{array}$ & $\begin{array}{l}\text { Ameliorates inflammation-induced insulin } \\
\text { resistance via lowering the pro-inflammatory } \\
\text { mediators as Nf-kb, ICAM-1, TNF- } \alpha \text { and } \\
\text { signaling pathways such as AMPK/mTOR }\end{array}$ & {$[17,92,93]$} \\
\hline $\begin{array}{c}\text { GLUT-4 } \\
\text { Expression/Localization }\end{array}$ & $\begin{array}{l}\text { Increase GLUT-4 expression/localization in } \\
\text { insulin-dependent tissues }\end{array}$ & [101-104] \\
\hline Lipid Metabolism & $\begin{array}{l}\text { Improve plasma lipid profile leading to a } \\
\text { lower rate of dyslipidemia-induced insulin } \\
\text { resistance }\end{array}$ & {$[121,123-126]$} \\
\hline Beta-cell Function & $\begin{array}{l}\text { Improve pancreatic beta-cell function via } \\
\text { several molecular pathways as neogenesis } \\
\text { induction and apoptosis inhibition }\end{array}$ & [133-136] \\
\hline $\begin{array}{l}\text { Insulin Signal } \\
\text { Transduction }\end{array}$ & $\begin{array}{l}\text { Amplify insulin signal transduction at various } \\
\text { steps such as Akt and IRS-1 phosphorylation }\end{array}$ & {$[100,127,128]$} \\
\hline ER Stress & $\begin{array}{l}\text { Attenuate ER stress thru mTOR signaling or } \\
\text { down-regulating the C/EBPB protein and } \\
\text { thereby decline ER stress-induced insulin } \\
\text { resistance }\end{array}$ & {$[18,135,145]$} \\
\hline
\end{tabular}

\section{Other potential molecular pathways}

In addition to the above described molecular pathways, some other molecular mechanisms may

be involved in GLP-1 dependent inulin sensitivity [88][146]. GLP-1 potentially suppress 
glucagon secretion which can lead to higher insulin sensitivity [146, 147]. Also, there is evidence suggesting GLP-1 can stimulate the expression and release of adipokines and adiponectin which have major roles in the insulin signaling pathways [88]. They can also potentially modulate the mitochondrial function and improves insulin resistance due to mitochondrial dysfunctions [148].

\section{Conclusion}

GLP-1 is one of the main peptides of the incretin family which modulates glucose homeostasis through several molecular pathways. The two GLP-1 pathways based therapies for the treatment of T2DM are GLP-1 receptor agonists and DPP-4 inhibitors. They act as antihyperglycemic agents through a variety of mechanisms including glucose dependent stimulation of insulin secretion, suppression of glucagon secretion, reduction of hepatic glucose output and suppression of appetite. These agents also increase insulin sensitivity via at least eight molecular pathways including oxidative stress, inflammation, ER stress, lipid metabolism, GLUT-4 expression/translocation, beta-cell function and insulin signaling pathways.

\section{Acknowledgment}

The authors are thankful to the "Research center of physiology, Semnan University of medical sciences (Semnan, Iran)" for providing technical supports.

\section{Conflict of Interests}

The authors declare that they have no conflict of interest in this study. 


\section{References}

1. Ogurtsova, K., et al., IDF Diabetes Atlas: Global estimates for the prevalence of diabetes for 2015 and 2040. Diabetes research and clinical practice, 2017. 128: p. 40-50.

2. Forbes, J.M. and M.E. Cooper, Mechanisms of diabetic complications. Physiological reviews, 2013. 93(1): p. 137-188.

3. Brownlee, M., The pathobiology of diabetic complications: a unifying mechanism. diabetes, 2005. 54(6): p. 1615-1625.

4. Volpe, C.M.O., et al., Cellular death, reactive oxygen species (ROS) and diabetic complications. Cell death \& disease, 2018. 9(2): p. 119.

5. Buraczynska, M., et al., The effect of Toll-like receptor 4 gene polymorphism on vascular complications in type 2 diabetes patients. Diabetes research and clinical practice, 2016. 116: p. 7-13.

6. Mattisson, I.Y., et al., Elevated markers of death receptor-activated apoptosis are associated with increased risk for development of diabetes and cardiovascular disease. EBioMedicine, 2017. 26: p. 187-197.

7. Jia, G., V.G. DeMarco, and J.R. Sowers, Insulin resistance and hyperinsulinaemia in diabetic cardiomyopathy. Nature Reviews Endocrinology, 2016. 12(3): p. 144.

8. Pop, A., et al., Insulin resistance is associated with all chronic complications in type 1 diabetes: 在 1 型糖尿病中胰岛素抵抗与所有的慢性并发症都相全. Journal of diabetes, 2016. 8(2): p. 220-228.

9. Patel, T.P., et al., Insulin resistance: an additional risk factor in the pathogenesis of cardiovascular disease in type 2 diabetes. Heart failure reviews, 2016. 21(1): p. 11-23.

10. Yaribeygi, H., et al., Effects of antidiabetic drugs on NLRP3 inflammasome activity, with a focus on diabetic kidneys. Drug discovery today, 2018.

11. Yaribeygi, H., et al., Antioxidative potential of antidiabetic agents: A possible protective mechanism against vascular complications in diabetic patients. Journal of cellular physiology, 2019. 234(3): p. 2436-2446.

12. Yaribeygi, H., et al., A review of the anti-inflammatory properties of antidiabetic agents providing protective effects against vascular complications in diabetes. Journal of cellular physiology, 2019. 234(6): p. 8286-8294.

13. DeFronzo, R.A., Combination therapy with GLP-1 receptor agonist and SGLT2 inhibitor. Diabetes, Obesity and Metabolism, 2017. 19(10): p. 1353-1362.

14. Trujillo, J.M., W. Nuffer, and S.L. Ellis, GLP-1 receptor agonists: a review of head-to-head clinical studies. Therapeutic advances in endocrinology and metabolism, 2015. 6(1): p. 19-28.

15. Prasad-Reddy, L. and D. Isaacs, A clinical review of GLP-1 receptor agonists: efficacy and safety in diabetes and beyond. Drugs in context, 2015. 4.

16. Cabou, C., et al., Brain glucagon-like peptide-1 regulates arterial blood flow, heart rate, and insulin sensitivity. Diabetes, 2008. 57(10): p. 2577-2587.

17. Guo, C., et al., Glucagon-like peptide 1 improves insulin resistance in vitro through antiinflammation of macrophages. Brazilian Journal of Medical and Biological Research, 2016. 49(12).

18. Jiang, Y., et al., GLP-1 improves adipocyte insulin sensitivity following induction of endoplasmic reticulum stress. Frontiers in pharmacology, 2018. 9: p. 1168.

19. Sandoval, D. and S.R. Sisley, Brain GLP-1 and insulin sensitivity. Molecular and cellular endocrinology, 2015. 418: p. 27-32.

20. Marathe, C.S., et al., Effects of GLP-1 and incretin-based therapies on gastrointestinal motor function. Experimental diabetes research, 2011. 2011.

21. Association, A.D., Diagnosis and classification of diabetes mellitus. Diabetes care, 2014. 37(Supplement 1): p. S81-S90.

22. de Faria Maraschin, J., Classification of diabetes, in Diabetes. 2013, Springer. p. 12-19. 
23. O'Neal, K.S., J.L. Johnson, and R.L. Panak, Recognizing and appropriately treating latent autoimmune diabetes in adults. Diabetes Spectrum, 2016. 29(4): p. 249-252.

24. Gailliot, M.T. and R.F. Baumeister, The physiology of willpower: Linking blood glucose to selfcontrol, in Self-Regulation and Self-Control. 2018, Routledge. p. 137-180.

25. Hall, J.E., Guyton and Hall textbook of medical physiology e-Book. 2015: Elsevier Health Sciences.

26. Chen, Y. and J. Lippincott-Schwartz, Selective visualization of GLUT4 storage vesicles and associated Rab proteins using IRAP-pHluorin, in Rab GTPases. 2015, Springer. p. 173-179.

27. Huang, S. and M.P. Czech, The GLUT4 glucose transporter. Cell metabolism, 2007. 5(4): p. 237252.

28. Moraes-Vieira, P.M., A. Saghatelian, and B.B. Kahn, GLUT4 expression in adipocytes regulates de novo lipogenesis and levels of a novel class of lipids with antidiabetic and anti-inflammatory effects. Diabetes, 2016. 65(7): p. 1808-1815.

29. Weiss, M., D.F. Steiner, and L.H. Philipson, Insulin biosynthesis, secretion, structure, and structure-activity relationships. 2014.

30. Koeppen, B.M. and B.A. Stanton, Berne and levy physiology e-book. 2017: Elsevier Health Sciences.

31. Rutter, G.A., et al., Pancreatic B-cell identity, glucose sensing and the control of insulin secretion. Biochem J, 2015. 466(2): p. 203-218.

32. Færch, K., et al., Insulin resistance is accompanied by increased fasting glucagon and delayed glucagon suppression in individuals with normal and impaired glucose regulation. Diabetes, 2016: p. db160240.

33. Kiselyov, V.V., et al., Harmonic oscillator model of the insulin and IGF1 receptors' allosteric binding and activation. Molecular systems biology, 2009. 5(1): p. 243.

34. Copps, K. and M. White, Regulation of insulin sensitivity by serine/threonine phosphorylation of insulin receptor substrate proteins IRS1 and IRS2. Diabetologia, 2012. 55(10): p. 2565-2582.

35. Ho, C.K., G. Sriram, and K.M. Dipple, Insulin sensitivity predictions in individuals with obesity and type II diabetes mellitus using mathematical model of the insulin signal transduction pathway. Molecular genetics and metabolism, 2016. 119(3): p. 288-292.

36. Wojtaszewski, J., et al., Insulin signaling and insulin sensitivity after exercise in human skeletal muscle. Diabetes, 2000. 49(3): p. 325-331.

37. Pessin, J.E. and A.R. Saltiel, Signaling pathways in insulin action: molecular targets of insulin resistance. The Journal of clinical investigation, 2000. 106(2): p. 165-169.

38. Chang, L., S.-H. Chiang, and A.R. Saltiel, Insulin signaling and the regulation of glucose transport. Molecular medicine, 2004. 10(7): p. 65.

39. Oh, Y. and H.-S. Jun, Effects of glucagon-like peptide-1 on oxidative stress and Nrf2 signaling. International journal of molecular sciences, 2017. 19(1): p. 26.

40. Donnelly, D., The structure and function of the glucagon-like peptide-1 receptor and its ligands. British journal of pharmacology, 2012. 166(1): p. 27-41.

41. Underwood, C.R., et al., Crystal structure of glucagon-like peptide-1 in complex with the extracellular domain of the glucagon-like peptide-1 receptor. Journal of Biological Chemistry, 2010. 285(1): p. 723-730.

42. Song, G., et al., Human GLP-1 receptor transmembrane domain structure in complex with allosteric modulators. Nature, 2017. 546(7657): p. 312.

43. Brubaker, P. and D. Drucker, Structure-function of the glucagon receptor family of $G$ proteincoupled receptors: the glucagon, GIP, GLP-1, and GLP-2 receptors. Receptors and Channels, 2002. 8(3-4): p. 179-188.

44. Wootten, D., et al., A hydrogen-bonded polar network in the core of the glucagon-like peptide1 receptor is a fulcrum for biased agonism: lessons from class $B$ crystal structures. Molecular pharmacology, 2016. 89(3): p. 335-347. 
45. Yaribeygi, H., et al., A review of the anti-inflammatory properties of antidiabetic agents providing protective effects against vascular complications in diabetes. Journal of cellular physiology, 2018.

46. Drucker, D.J. and M.A. Nauck, The incretin system: glucagon-like peptide-1 receptor agonists and dipeptidyl peptidase-4 inhibitors in type 2 diabetes. The Lancet, 2006. 368(9548): p. 16961705.

47. Islam, M., Insulinotropic Effect of Herbal Drugs for Management of Diabetes Mellitus: A Congregational Approach. Biosens J, 2016. 5(142): p. 2.

48. Meier, J.J., GLP-1 receptor agonists for individualized treatment of type 2 diabetes mellitus. Nature Reviews Endocrinology, 2012. 8(12): p. 728.

49. Baggio, L.L. and D.J. Drucker, Biology of incretins: GLP-1 and GIP. Gastroenterology, 2007. 132(6): p. 2131-2157.

50. Scott, K.A. and T.H. Moran, The GLP-1 agonist exendin-4 reduces food intake in nonhuman primates through changes in meal size. American Journal of Physiology-Regulatory, Integrative and Comparative Physiology, 2007. 293(3): p. R983-R987.

51. Ding, X., et al., Exendin-4, a glucagon-like protein-1 (GLP-1) receptor agonist, reverses hepatic steatosis in ob/ob mice. Hepatology, 2006. 43(1): p. 173-181.

52. Association, A.D., 2. Classification and diagnosis of diabetes: standards of medical care in diabetes-2018. Diabetes Care, 2018. 41(Supplement 1): p. S13-S27.

53. Ahren, B., DPP-4 inhibitors. Best Practice \& Research Clinical Endocrinology \& Metabolism, 2007. 21(4): p. 517-533.

54. Brubaker, P.L., The Glucagon-Like Peptides. Annals of the New York Academy of Sciences, 2006. 1070(1): p. 10-26.

55. Sun, A.-I., et al., Dipeptidyl peptidase-IV is a potential molecular biomarker in diabetic kidney disease. Diabetes and Vascular Disease Research, 2012. 9(4): p. 301-308.

56. Parlevliet, E.T., et al., GLP-1 treatment reduces endogenous insulin resistance via activation of central GLP-1 receptors in mice fed a high-fat diet. American Journal of PhysiologyEndocrinology and Metabolism, 2010. 299(2): p. E318-E324.

57. Meloni, A., et al., GLP-1 receptor activated insulin secretion from pancreatic B-cells: mechanism and glucose dependence. Diabetes, Obesity and Metabolism, 2013. 15(1): p. 1527.

58. Inaba, W., et al., Effects of long-term treatment with the dipeptidyl peptidase-4 inhibitor vildagliptin on islet endocrine cells in non-obese type 2 diabetic Goto-Kakizaki rats. European journal of pharmacology, 2012. 691(1-3): p. 297-306.

59. Jones, B., et al., Control of insulin secretion by GLP-1. Peptides, 2018. 100: p. 75-84.

60. Kirk, R.K., et al., Immunohistochemical assessment of glucagon-like peptide 1 receptor (GLP$1 R$ ) expression in the pancreas of patients with type 2 diabetes. Diabetes, Obesity and Metabolism, 2017. 19(5): p. 705-712.

61. Carlessi, R., et al., GLP-1 receptor signalling promotes 6 -cell glucose metabolism via mTORdependent HIF-1 $\alpha$ activation. Scientific reports, 2017. 7(1): p. 2661.

62. Rowlands, J., et al., Pleiotropic Effects of GLP-1 and analogs on cell signaling, metabolism and function. Frontiers in endocrinology, 2018. 9: p. 672.

63. Yaribeygi, H., et al., Oxidative stress induces renal failure: A review of possible molecular pathways. Journal of cellular biochemistry, 2018. 119(4): p. 2990-2998.

64. Yaribeygi, H., S.L. Atkin, and A. Sahebkar, Mitochondrial dysfunction in diabetes and the regulatory roles of antidiabetic agents on the mitochondrial function. Journal of cellular physiology, 2019. 234(6): p. 8402-8410.

65. Yaribeygi, H., et al., Insulin resistance: Review of the underlying molecular mechanisms. Journal of cellular physiology, 2019. 234(6): p. 8152-8161.

66. Hurrle, S. and W.H. Hsu, The etiology of oxidative stress in insulin resistance. biomedical journal, 2017. 40(5): p. 257-262. 
67. Furukawa, S., et al., Increased oxidative stress in obesity and its impact on metabolic syndrome. The Journal of clinical investigation, 2017. 114(12): p. 1752-1761.

68. Elmarakby, A.A. and J.C. Sullivan, Relationship between oxidative stress and inflammatory cytokines in diabetic nephropathy. Cardiovascular therapeutics, 2012. 30(1): p. 49-59.

69. Yaribeygi, H., S.L. Atkin, and A. Sahebkar, A review of the molecular mechanisms of hyperglycemia-induced free radical generation leading to oxidative stress. Journal of cellular physiology, 2019. 234(2): p. 1300-1312.

70. Yaribeygi, H., et al., Sodium-glucose cotransporter inhibitors and oxidative stress: An update. Journal of cellular physiology, 2019. 234(4): p. 3231-3237.

71. Laviola, L., et al., Glucagon-like peptide-1 counteracts oxidative stress-dependent apoptosis of human cardiac progenitor cells by inhibiting the activation of the c-Jun N-terminal protein kinase signaling pathway. Endocrinology, 2012. 153(12): p. 5770-5781.

72. Cai, X., et al., GLP-1 treatment protects endothelial cells from oxidative stress-induced autophagy and endothelial dysfunction. International journal of biological sciences, 2018. 14(12): p. 1696.

73. Deng, C., et al., Liraglutide activates the Nrf2/HO-1 antioxidant pathway and protects brain nerve cells against cerebral ischemia in diabetic rats. Computational intelligence and neuroscience, 2018. 2018.

74. Uruno, A., et al., The Keap1-Nrf2 system prevents onset of diabetes mellitus. Molecular and cellular biology, 2013. 33(15): p. 2996-3010.

75. Holmström, K.M., et al., Nrf2 impacts cellular bioenergetics by controlling substrate availability for mitochondrial respiration. Biology open, 2013. 2(8): p. 761-770.

76. Fernández-Millán, E., et al., Glucagon-like peptide-1 improves beta-cell antioxidant capacity via extracellular regulated kinases pathway and Nrf2 translocation. Free Radical Biology and Medicine, 2016. 95: p. 16-26.

77. Puddu, A., et al., An emerging role of glucagon-like peptide-1 in preventing advancedglycation-end-product-mediated damages in diabetes. Mediators of inflammation, 2013. 2013.

78. Tomas, E., V. Stanojevic, and J.F. Habener, GLP-1-derived nonapeptide GLP-1 (28-36) amide targets to mitochondria and suppresses glucose production and oxidative stress in isolated mouse hepatocytes. Regulatory peptides, 2011. 167(2-3): p. 177-184.

79. Patel, V., et al., Combination of omeprazole with GLP-1 agonist therapy improves insulin sensitivity and antioxidant activity in liver in type 1 diabetic mice. Pharmacological Reports, 2013. 65(4): p. 927-936.

80. Okada, K., et al., Effects of treatment with liraglutide on oxidative stress and cardiac natriuretic peptide levels in patients with type 2 diabetes mellitus. Endocrine, 2014. 47(3): p. 962-964.

81. Rizzo, M., et al., Liraglutide reduces oxidative stress and restores heme oxygenase-1 and ghrelin levels in patients with type 2 diabetes: a prospective pilot study. The journal of clinical endocrinology \& metabolism, 2015. 100(2): p. 603-606.

82. Yaribeygi, H., S.L. Atkin, and A. Sahebkar, Interleukin-18 and diabetic nephropathy: A review. Journal of cellular physiology, 2019. 234(5): p. 5674-5682.

83. De Luca, C. and J.M. Olefsky, Inflammation and insulin resistance. FEBS letters, 2008. 582(1): p. 97-105.

84. Fantuzzi, G., Adipose tissue, adipokines, and inflammation. Journal of Allergy and clinical immunology, 2005. 115(5): p. 911-919.

85. Tilg, H. and A.R. Moschen, Inflammatory mechanisms in the regulation of insulin resistance. Molecular medicine, 2008. 14(3-4): p. 222-231.

86. Hattori, Y., et al., RETRACTED ARTICLE: A glucagon-like peptide-1 (GLP-1) analogue, liraglutide, upregulates nitric oxide production and exerts anti-inflammatory action in endothelial cells. Diabetologia, 2010. 53(10): p. 2256. 
87. Krasner, N.M., et al., Glucagon-like peptide-1 (GLP-1) analog liraglutide inhibits endothelial cell inflammation through a calcium and AMPK dependent mechanism. Plos one, 2014. 9(5): p. e97554.

88. Hosaka, T., et al., Exendin-4, a GLP-1 receptor agonist, directly induces adiponectin expression through protein kinase A pathway and prevents inflammatory adipokine expression. Biochemical and biophysical research communications, 2009. 390(3): p. 613-618.

89. Lee, Y.-S. and H.-S. Jun, Anti-inflammatory effects of GLP-1-based therapies beyond glucose control. Mediators of inflammation, 2016. 2016.

90. Shiraki, A., et al., The glucagon-like peptide 1 analog liraglutide reduces TNF- $\alpha$-induced oxidative stress and inflammation in endothelial cells. Atherosclerosis, 2012. 221(2): p. 375382.

91. Kodera, R., et al., Glucagon-like peptide-1 receptor agonist ameliorates renal injury through its anti-inflammatory action without lowering blood glucose level in a rat model of type 1 diabetes. Diabetologia, 2011. 54(4): p. 965-978.

92. Zheng, W., et al., Dipeptidyl-peptidase 4 inhibitor sitagliptin ameliorates hepatic insulin resistance by modulating inflammation and autophagy in ob/ob mice. International journal of endocrinology, 2018. 2018.

93. Zhuge, F., et al., DPP-4 inhibition by linagliptin attenuates obesity-related inflammation and insulin resistance by regulating M1/M2 macrophage polarization. Diabetes, 2016. 65(10): p. 2966-2979.

94. Shan, W.-f., et al., Effects of GLUT4 expression on insulin resistance in patients with advanced liver cirrhosis. Journal of Zhejiang University SCIENCE B, 2011. 12(8): p. 677.

95. Harriet Wallberg-Henriksson, J.R.Z., GLUT4: a key player regulating glucose homeostasis? Insights from transgenic and knockout mice. Molecular membrane biology, 2001. 18(3): p. 205-211.

96. Sancho, V., et al., Effects of glucagon-like peptide-1 and exendins on kinase activity, glucose transport and lipid metabolism in adipocytes from normal and type-2 diabetic rats. Journal of molecular endocrinology, 2005. 35(1): p. 27-38.

97. Sancho, V., et al., The action of GLP-1 and exendins upon glucose transport in normal human adipocytes, and on kinase activity as compared to morbidly obese patients. International journal of molecular medicine, 2007. 19(6): p. 961-966.

98. Arnés, L., et al., Characteristics of GLP-1 and exendins action upon glucose transport and metabolism in type 2 diabetic rat skeletal muscle. International journal of molecular medicine, 2008. 22(1): p. 127-132.

99. Villanueva-Peñacarrillo, M.L., et al., Effect of GLP-1 treatment on GLUT2 and GLUT4 expression in type 1 and type 2 rat diabetic models. Endocrine, 2001. 15(2): p. 241-248.

100. Wang, Y., et al., Regulation of glucose transporters and hexose uptake in 3T3-L1 adipocytes: glucagon-like peptide-1 and insulin interactions. Journal of molecular endocrinology, 1997. 19(3): p. 241-248.

101. Green, C.J., et al., Glucagon like peptide-1-induced glucose metabolism in differentiated human muscle satellite cells is attenuated by hyperglycemia. PloS one, 2012. 7(8): p. e44284.

102. Andreozzi, F., et al., The GLP-1 receptor agonists exenatide and liraglutide activate Glucose transport by an AMPK-dependent mechanism. Journal of translational medicine, 2016. 14(1): p. 229.

103. Li, Z., et al., Liraglutide enhances glucose transporter 4 translocation via regulation of AMPactivated protein kinase signaling pathways in mouse skeletal muscle cells. Metabolism, 2014. 63(8): p. 1022-1030.

104. Giannocco, G., et al., Dipeptidyl peptidase IV inhibition upregulates GLUT4 translocation and expression in heart and skeletal muscle of spontaneously hypertensive rats. European journal of pharmacology, 2013. 698(1-3): p. 74-86. 
105. Zheng, S., et al., Associations of lipid profiles with insulin resistance and $B$ cell function in adults with normal glucose tolerance and different categories of impaired glucose regulation. PloS one, 2017. 12(2): p. e0172221.

106. Kawamoto, R., et al., Relationships between lipid profiles and metabolic syndrome, insulin resistance and serum high molecular adiponectin in Japanese community-dwelling adults. Lipids in health and disease, 2011. 10(1): p. 79.

107. Kahn, S.E., R.L. Hull, and K.M. Utzschneider, Mechanisms linking obesity to insulin resistance and type 2 diabetes. Nature, 2006. 444(7121): p. 840.

108. Polsky, S. and S.L. Ellis, Obesity, insulin resistance, and type 1 diabetes mellitus. Current Opinion in Endocrinology, Diabetes and Obesity, 2015. 22(4): p. 277-282.

109. Tangvarasittichai, S., Oxidative stress, insulin resistance, dyslipidemia and type 2 diabetes mellitus. World journal of diabetes, 2015. 6(3): p. 456.

110. Farr, S., J. Taher, and K. Adeli, Glucagon-like peptide-1 as a key regulator of lipid and lipoprotein metabolism in fasting and postprandial states. Cardiovascular \& Haematological Disorders-Drug Targets (Formerly Current Drug Targets-Cardiovascular \& Hematological Disorders), 2014. 14(2): p. 126-136.

111. Lutz, T.A. and E. Osto, Glucagon-like peptide-1, glucagon-like peptide-2, and lipid metabolism. Current opinion in lipidology, 2016. 27(3): p. 257-263.

112. J Patel, V., et al., Effect of GLP-1 based therapies on diabetic dyslipidemia. Current diabetes reviews, 2014. 10(4): p. 238-250.

113. Shen, Y., et al., MicroRNA profile changes in liraglutide treated steatotic HepG2 cells. Int J Clin Exp Med, 2017. 10(3): p. 4856-4864.

114. Capuani, B., et al., Glucagon Like Peptide 1 and MicroRNA in Metabolic Diseases: Focusing on GLP1 Action on miRNAs. Frontiers in endocrinology, 2018. 9.

115. Guo, J., et al., Reduced miR-200b and miR-200c expression contributes to abnormal hepatic lipid accumulation by stimulating JUN expression and activating the transcription of srebp1. Oncotarget, 2016. 7(24): p. 36207.

116. Wei, J., et al., MiR-338 controls BPA-triggered pancreatic islet insulin secretory dysfunction from compensation to decompensation by targeting Pdx-1. The FASEB Journal, 2017. 31(12): p. 5184-5195.

117. Akaslan, S.B., et al., Effects of sitagliptin on nonalcoholic fatty liver disease in diet-induced obese rats. Metabolic syndrome and related disorders, 2013. 11(4): p. 243-250.

118. Kato, H., et al., Effect of sitagliptin on intrahepatic lipid content and body fat in patients with type 2 diabetes. Diabetes research and clinical practice, 2015. 109(1): p. 199-205.

119. Mulvihill, E.E., Regulation of intestinal lipid and lipoprotein metabolism by the proglucagonderived peptides glucagon like peptide 1 and glucagon like peptide 2. Current opinion in lipidology, 2018. 29(2): p. 95.

120. Gastaldelli, A. and G. Marchesini, Time for Glucagon like peptide-1 receptor agonists treatment for patients with NAFLD? Journal of hepatology, 2016. 64(2): p. 262-264.

121. Ejarque, M., et al., Role of adipose tissue GLP-1R expression in metabolic improvement after bariatric surgery in patients with type 2 diabetes. Scientific reports, 2019. 9(1): p. 6274.

122. Parlevliet, E.T., et al., GLP-1 receptor activation inhibits VLDL production and reverses hepatic steatosis by decreasing hepatic lipogenesis in high-fat-fed APOE* 3-Leiden mice. PloS one, 2012. 7(11): p. e49152.

123. Cani, P.D., et al., Improvement of glucose tolerance and hepatic insulin sensitivity by oligofructose requires a functional glucagon-like peptide 1 receptor. Diabetes, 2006. 55(5): p. 1484-1490.

124. Baumeier, C., et al., Elevated hepatic DPP4 activity promotes insulin resistance and nonalcoholic fatty liver disease. Molecular metabolism, 2017. 6(10): p. 1254-1263. 
125. Silva Júnior, W.S., et al., Dipeptidyl Peptidase 4 Activity Is Related to Body Composition, Measures of Adiposity, and Insulin Resistance in Subjects with Excessive Adiposity and Different Degrees of Glucose Tolerance. Journal of diabetes research, 2019. 2019.

126. Deacon, C.F., Physiology and pharmacology of DPP-4 in glucose homeostasis and the treatment of type 2 diabetes. Frontiers in endocrinology, 2019. 10: p. 80.

127. Gao, H., et al., GLP-1 amplifies insulin signaling by up-regulation of IRB, IRS-1 and Glut4 in 3T3L1 adipocytes. Endocrine, 2007. 32(1): p. 90-95.

128. Kawamori, D., et al., GLP-1 signalling compensates for impaired insulin signalling in regulating beta cell proliferation in BIRKO mice. Diabetologia, 2017. 60(8): p. 1442-1453.

129. Cernea, S. and M. Dobreanu, Diabetes and beta cell function: from mechanisms to evaluation and clinical implications. Biochemia medica: Biochemia medica, 2013. 23(3): p. 266-280.

130. Maedler, K. and M. Donath, 6-Cells in type 2 diabetes: a loss of function and mass. Hormone Research in Paediatrics, 2004. 62(Suppl. 3): p. 67-73.

131. Page, K.A. and T. Reisman, Interventions to preserve beta-cell function in the management and prevention of type 2 diabetes. Current diabetes reports, 2013. 13(2): p. 252-260.

132. Wajchenberg, B.L., Clinical approaches to preserve B-cell function in diabetes, in The Islets of Langerhans. 2010, Springer. p. 515-535.

133. Tews, D., et al., Anti-apoptotic action of exendin-4 in INS-1 beta cells: comparative protein pattern analysis of isolated mitochondria. Hormone and metabolic research, 2009. 41(04): p. 294-301.

134. Kim, M.-H., et al., EX4 stabilizes and activates Nrf2 via PKCD, contributing to the prevention of oxidative stress-induced pancreatic beta cell damage. Toxicology and applied pharmacology, 2017. 315: p. 60-69.

135. Shimoda, M., et al., The human glucagon-like peptide-1 analogue liraglutide preserves pancreatic beta cells via regulation of cell kinetics and suppression of oxidative and endoplasmic reticulum stress in a mouse model of diabetes. Diabetologia, 2011. 54(5): p. 10981108.

136. Gedulin, B.R., et al., Exenatide (exendin-4) improves insulin sensitivity and B-cell mass in insulin-resistant obese fa/fa Zucker rats independent of glycemia and body weight. Endocrinology, 2005. 146(4): p. 2069-2076.

137. Caporarello, N., et al., Insulin receptor signaling and glucagon-like peptide 1 effects on pancreatic beta cells. PloS one, 2017. 12(8): p. e0181190.

138. Buteau, J., GLP-1 signaling and the regulation of pancreatic B-cells mass/function. Avances en Diabetología, 2011. 27(1): p. 3-8.

139. Hao, T., et al., Glucagon-like peptide 1 receptor agonist ameliorates the insulin resistance function of islet $B$ cells via the activation of PDX-1/JAK signaling transduction in C57/BL6 mice with high-fat diet-induced diabetes. International journal of molecular medicine, 2017. 39(4): p. 1029-1036.

140. Zummo, F.P., et al., Glucagon-like peptide 1 protects pancreatic B-cells from death by increasing autophagic flux and restoring lysosomal function. Diabetes, 2017. 66(5): p. 12721285.

141. Akarte, A.S., B. Srinivasan, and S. Gandhi, Vildagliptin selectively ameliorates GLP-1, GLUT4, SREBP-1c mRNA levels and stimulates b-cell proliferation resulting in improved glucose homeostasis in rats with streptozotocin-induced diabetes. Journal of Diabetes and its Complications, 2012. 26(4): p. 266-274.

142. Ron, D. and P. Walter, Signal integration in the endoplasmic reticulum unfolded protein response. Nature reviews Molecular cell biology, 2007. 8(7): p. 519.

143. Özcan, U., et al., Endoplasmic reticulum stress links obesity, insulin action, and type 2 diabetes. Science, 2004. 306(5695): p. 457-461.

144. Szegezdi, E., et al., Mediators of endoplasmic reticulum stress-induced apoptosis. EMBO reports, 2006. 7(9): p. 880-885. 
145. Shimizu, S., et al., DPP4 inhibitor vildagliptin preserves-cell mass through amelioration of endoplasmic reticulum stress in C/EBPB transgenic mice. Journal of molecular endocrinology, 2012. 49(2): p. 125.

146. Ramracheya, R., et al., GLP-1 suppresses glucagon secretion in human pancreatic alpha-cells by inhibition of P/Q-type Ca2+ channels. Physiological reports, 2018. 6(17): p. e13852.

147. Færch, K., et al., Insulin resistance is accompanied by increased fasting glucagon and delayed glucagon suppression in individuals with normal and impaired glucose regulation. Diabetes, 2016. 65(11): p. 3473-3481.

148. Katayama, T., et al., The Activation of Glucagon-Like Peptide-1 Improves the Mitochondrial Abnormalities in Skeletal Muscle and Exercise Intolerance in Heart Failure Mice. Journal of Cardiac Failure, 2016. 22(9): p. S162. 